\title{
Engineering and Advanced Digitalization of Photonic Structures with Bound Field in the Continuum
}

\author{
N. Prodanović, V. Milanović and J. Radovanović* \\ School of Electrical Engineering, University of Belgrade, Belgrade, Serbia
}

\begin{abstract}
We describe a method for generation of complex optical potentials which support a bound state of the electric field in continuous part of the spectrum. It is based on deep analogy between quantum mechanical and electromagnetic phenomena and relies on the application of supersymmetric quantum mechanics to generate a smoothly varying complex optical potential, together with the corresponding electric field function for the (single) localized state. However, the obtained potential profile is generally a strongly oscillating function which requires additional processing to make it suitable for practical realization. With this goal in mind, i.e. the construction of a realizable photonic crystal with complex permittivity which supports one bound state in continuum, we have developed an original scheme of digital grading. It approximates the values of the complex relative permittivity in such manner that the final structure may be realized by assembling layers of homogeneous materials.
\end{abstract}

PACS numbers: 11.30.Pb, 03.65.Ge, 42.25.Bs

\section{Introduction}

Supersymmetric quantum mechanics (SUSYQM) is a method that can be used to obtain operators which are almost isospectral, except that one of them has an additional bound state in continuum. Von Neumann and Wigner [1] found that the Schrödinger equation may have regular solutions which represent bound states in the continuum part of the spectrum, and Herrick and Stillinger [2-4] have shown that bound states in continuum may exist in atoms and molecules. However, while the existence of normalizable eigensolutions for non-local potentials is rather well explored [5], a systematic approach for local potentials is still missing.

In addition, there is a close analogy between quantum mechanical and electromagnetic phenomena. In Ref. [6], the existence of bound states in radiation continuum is illustrated on the example of two parallel gratings and two arrays of thin parallel cylinders. Also, in Ref. [7]. it is shown that photonic crystals with defects may have localized states in the continuum part of the spectrum.

In this paper, we start from the modified form of the Helmholtz equation for the electric field, which is analogous to the Schrödinger equation (and so are their general solutions), in order to construct complex permittivity functions which correspond to the selected initial one in terms of electric field spectrum. Each of the newly obtained complex permittivity profiles supports one and only one localized normalizable function of the electric field in the continuum part of the spectrum. We first give a short description of the SUSY procedure, details of which can be found in $[8,9]$ applied to a quantum mechanical problem, and then implement it to the

* corresponding author; e-mail: radovanovic@etf.bg.ac.rs case of flat permittivity. Finally, we present somewhat non-standard digital grading approximation of generated complex permittivity and numerical solution for the electrical field function corresponding to it, with satisfactory similarity to the original solution.

\section{Theoretical framework}

Consider electromagnetic (EM) waves propagating along the $x$-direction, through a material which is linear and non-homogeneous in this direction and with constant magnetic permeability $\mu=1$. Only linearly polarized TE modes are considered, i.e. $\boldsymbol{E}=E \boldsymbol{e}_{y}$ which are also monochromatic, i.e. $E(t)=E \mathrm{e}^{\mathrm{i} \omega_{0} t}$. Starting from the usual form of the Helmholtz equation and solving it by separation of variables $E(x, y, z, t)=E(x) E(y) E(z) \mathrm{e}^{\mathrm{i} \omega_{0} t}$ one can show that $E(z)=E \mathrm{e}^{\mathrm{i} \beta z}, E(y)=$ const, and most importantly

$$
\frac{\mathrm{d}^{2} E(x)}{\mathrm{d} x^{2}}+k_{0}^{2} \varepsilon(x) E(x)=\beta^{2} E(x),
$$

where $k_{0}=\omega_{0} / c$.

Equation (1) is analogous to the Schrödinger equation $-\frac{\hbar^{2}}{2 m} \frac{\mathrm{d}^{2} \psi(x)}{\mathrm{d} x^{2}}+U(x) \psi(x)=E \psi(x)$. As described in Ref. [9], the idea is to construct isospectral operator $-\frac{\mathrm{d}^{2}}{\mathrm{~d} x^{2}}-k_{0}^{2} \varepsilon_{s s}(x, \rho, C)$, with complex constants $\rho$ and $C$, by using standard SUSY procedure. The derived formula, as given in [9], reads

$$
\begin{aligned}
& \varepsilon_{\mathrm{ss}}(x)=\varepsilon(x)+\frac{2}{k_{0}^{2}} \frac{\mathrm{d}^{2}}{\mathrm{~d} x^{2}}[\ln (\rho \\
& \left.\left.\quad+\int_{(x)} E_{\beta}^{2}(x)\left[1+C \int_{(x)} \frac{\mathrm{d} x}{E_{\beta}^{2}(x)}\right]^{2} \mathrm{~d} x\right)\right],
\end{aligned}
$$

where $E_{\beta}(x)$ is the eigenstate corresponding to a chosen eigenvalue $\beta^{2}$. Thus, the permittivity given by expression (2) is isospectral to $\varepsilon(x)$, and the additional (so-called 
supersymetric) eigenstate for a chosen eigenvalue, which was used for the SUSY procedure [9], is given by

$$
\begin{aligned}
& E_{\beta \mathrm{SS}}(x) \\
& =C_{\beta \mathrm{sS}} \frac{E_{\beta}(x)\left[1+C \int_{(x)} \frac{\mathrm{d} x}{E_{\beta}^{2}(x)}\right]}{\rho+\int_{(x)} E_{\beta}^{2}(x)\left[1+C \int_{(x)} \frac{\mathrm{d} x}{E_{\beta}^{2}(x)}\right]^{2} \mathrm{~d} x} .
\end{aligned}
$$

It can be proven that if the initial eigenvalue $\beta^{2}$ corresponds to the continuous part of the spectrum, then its supersymetric eigenstate is strictly a bound state in continuum, provided that the appropriate choice of parameters $\rho$ and $C$ is made [10].

First, we need to choose an initial permittivity with continuous spectra for generating a new (required) one with bound state in continuum. The simplest approach is to choose a "flat" initial permittivity $\varepsilon$. The general solution of (1) is then given by $E_{k}(x)=\sin (k x)+C \cos (k x)$ where $k=\sqrt{k_{0}^{2} \varepsilon-\beta^{2}}$. Clearly, the spectrum of the flat permittivity is completely continuous for $\beta^{2}<k_{0}^{2} \varepsilon$. The aim is to employ the SUSY transform to find a complex permittivity that accommodates a bound state at the selected eigenvalue. The final electric field function can be expressed as

$$
\sim \frac{E_{\mathrm{SS}}(x)}{\rho+\frac{x}{2}-\frac{\sin (2 k x)}{4 k}-\frac{C \cos ^{2}(k x)}{k}+C^{2}\left[\frac{x}{2}+\frac{\sin (2 k x)}{4 k}\right]},
$$

while the corresponding supersymmetric permittivity is given by

$$
\begin{gathered}
\varepsilon_{\mathrm{SS}}(x)=\varepsilon(x)+\frac{2}{k_{0}^{2}} \frac{\mathrm{d}^{2}}{\mathrm{~d} x^{2}}\left[\operatorname { l n } \left(\rho+\frac{x}{2}\right.\right. \\
\left.\left.-\frac{\sin (2 k x)}{4 k}-\frac{C \cos ^{2}(k x)}{k}+C^{2}\left(\frac{x}{2}+\frac{\sin (2 k x)}{4 k}\right)\right)\right] .
\end{gathered}
$$

The discussion on selecting the appropriate values of parameters $\rho$ and $C$ is given in Ref. [9], and in the following numerical example one such choice is illustrated.

The ultimate goal is to enable practical realization, i.e. to construct a photonic crystal with permittivity $\varepsilon(x)$, given by expression (5), which supports the bound state in continuum. This can be accomplished by applying the digital grading approximation directly to the supersymmetric permittivity and then composing very accurately the obtained digitally graded function.

\section{Digital grading of the complex relative permittivity}

The digital grading approximation of a complex function is uncommon, therefore it will be explained here in detail, assuming that the reader is familiar with the standard digital grading approximation of real profiles.

The first step is to define the segment of the structure that will undergo digital grading. Here we select a domain symmetric around zero, as both the real and the imaginary part of the final function are almost symmet- ric or antisymmetric. The peripheral parts of the function are "flattened" by taking the average values within particular areas. It can be shown that the value of the initial (constant) relative permittivity may be taken as a satisfactory estimate of that flat outer part of the supersymmetric permittivity. Such averaging of the peripheral area implies that the corresponding field will not be exactly bound, but it will oscillate with sufficiently small amplitude and frequency.

The second step involves the application of digital grading formalism to both the real and the imaginary part of relative permittivity in previously defined central area, in the usual manner, as presented in [11], with a few modifications.

In the procedure applied here, three levels (values) are used to approximate the final function, instead of only two values as in Ref. [11]. This improves the accuracy of the approximation, but complicates the construction of the obtained structure by increasing the number of constituent materials. Supersymmetric relative permittivity is a strongly oscillating function around some average value that is almost equal to the value in bulk or outside of the digitally graded area, so grading with only two levels gives poor results and cannot be utilized. Hence, an additional (medium level) is introduced as the average value of the function outside of the digitally graded area, namely as in the "flattened" area. The higher and the lower level are defined as in [11], as the extrema of the function over the entire domain.

As described in [11], the complete domain is divided into intervals which are then individually approximated with two different level combinations: the medium and the high level or the medium and the low level as shown in Fig. 1. Those intervals will be from now on referred to as "common cells". Thus, the common cell represent a standard interval where the graded approximate function (both, the real and the imaginary part) has only two values or levels. The average value of $\varepsilon_{\mathrm{ss}}(x)$ determines the pair of levels which is selected for each common cell. In more detail, the medium and the high level are used to describe a particular cell if the average value of $\varepsilon_{\mathrm{ss}}(x)$ within it is greater than the medium level, while the medium and the low level combination is used in the opposite case.

However, the smallest homogeneous units intended for deposition are not the segments occupied by individual levels in each common cell, but the subcells which will be introduced later.

The width of each level within a particular cell depends on the value of the integral $S=\int_{\text {Cell }}\left|\varepsilon_{\mathrm{ss}}(x)-\varepsilon_{\text {med }}\right| \mathrm{d} x$ where $\varepsilon_{\text {med }}$ is the value of one of the two levels appearing in a particular cell used as reference. In this work, by definition, it is always assumed that $\varepsilon_{\text {med }}$ is the value corresponding to the medium level as it is the only level present in each cell. The width of a non medium level $w_{\text {high/low }}$, which is either high or low for a specific cell, is defined by the next relation $w_{\text {high } / \text { low }}=$ $\frac{S}{\left|\varepsilon_{\text {med }}-\varepsilon_{\text {high } / \text { low }}\right|}$. The width of the medium level is then 


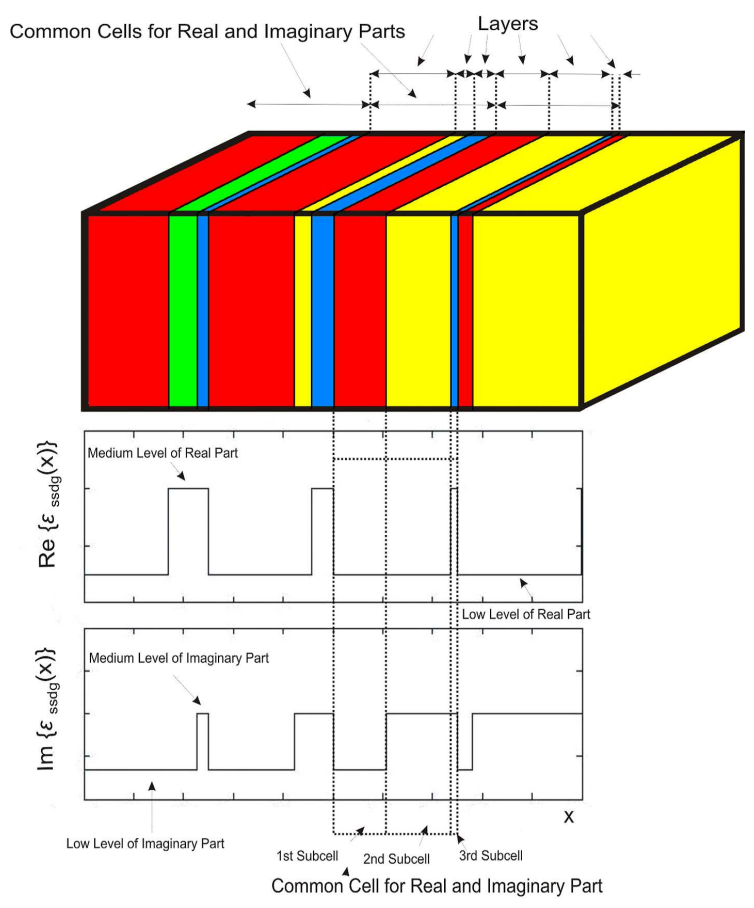

Fig. 1. Realization of the complex digitally graded function $\varepsilon_{\text {ssdg. }}$. Within one common cell interval of digitally graded real and imaginary part, three subcells-subintervals are marked by dashed lines. 1st subcell (red) is characterized by a combination of low level real part and low level imaginary part. 2nd subcell (yellow) is characterized by a combination of low level real part and medium level imaginary part. 3rd subcell (blue) is characterized by a combination of medium level real part and medium level imaginary part.

$w_{\text {med }}=d-w_{\text {high } / \text { low }}$, where $d$ represents the width of the cells.

Finally, in the third step previously obtained real and imaginary digitally graded functions are used to compose the complex digitally graded structure. The complex levels are introduced as combinations of real and imaginary levels. By combining three real and three imaginary levels, nine different complex levels are obtained. If two-level digital grading approximation was used then such combining would provide $2 \times 2$ complex levels.

This procedure entails the division of each common cell into subcells so that exactly one complex level can be assigned to each subcell, as shown in Fig. 1. The whole structure can thus be constructed in practice by deposition of layers of different materials corresponding to each subcell. This implies that each subcell consists of one specific layer of suitable material chosen from the set of nine different materials if three-level digital grading is considered, or from the set of four materials if conventional two-level digital grading is in use.

\section{Numerical examples and discussion}

Depending on the selection of values of $\beta, \varepsilon$ and $\lambda_{0}$ for the flat optical potential, various supersymmetric optical potentials are obtained. One can take for example: $k=$ $3 \mathrm{~mm}^{-1}, \varepsilon=5, \lambda_{0}=630 \mu \mathrm{m}$, where $k_{0}=\omega_{0} / c=2 \pi / \lambda_{0}$ denotes the wave number outside of the digitally graded area (in the homogenous part, which can be considered in the limit $x \rightarrow \pm \infty$ ), with relative permittivity $\varepsilon_{\mathrm{ss}}=\varepsilon$ as $\Theta_{\mathrm{ss}}(x \rightarrow \pm \infty)=-k_{0}^{2} \varepsilon$. The wave number $k$ defines the eigenvalue $\beta$ for which the SUSY formalism is employed. The remaining parameters are then calculated as: $k_{0}=10 \mathrm{~mm}^{-1}, \beta^{2}=k_{0}^{2} \varepsilon-k^{2}=491 \mathrm{~mm}^{-2}$. In addition, $C$ and $\rho$ are defined so that the supersymmetric eigenfunction is normalizable: $C=3+3 \mathrm{i}, \rho=9 \mathrm{~mm}$. In the numerical example treated here, three real and three imaginary levels of relative permittivity are calculated:

$$
\begin{aligned}
& \operatorname{Re}\left(\varepsilon_{\text {high }}\right)=5.1513, \quad \operatorname{Im}\left(\varepsilon_{\text {high }}\right)=0.15871, \\
& \operatorname{Re}\left(\varepsilon_{\text {med }}\right)=5, \quad \operatorname{Im}\left(\varepsilon_{\text {med }}\right)=0, \\
& \operatorname{Re}\left(\varepsilon_{\text {low }}\right)=4.9318, \quad \operatorname{Im}\left(\varepsilon_{\text {low }}\right)=-0.15565 .
\end{aligned}
$$

The combinations of these levels yield nine different homogeneous materials whose relative permittivity values are

$$
\begin{aligned}
& \varepsilon_{\mathrm{ssdg} 1}=5.1513+\mathrm{i}^{*} 0.15871, \quad \varepsilon_{\mathrm{ssdg} 2}=5.1513 \\
& \varepsilon_{\mathrm{ssdg} 3}=5.1513-\mathrm{i}^{*} 0.15565, \quad \varepsilon_{\mathrm{ssdg} 4}=5+\mathrm{i}^{*} 0.15871 \\
& \varepsilon_{\mathrm{ssdg} 5}=5, \quad \varepsilon_{\mathrm{ssdg} 6}=5-\mathrm{i}^{*} 0.15565 \\
& \varepsilon_{\mathrm{ssdg} 7}=4.9318+\mathrm{i}^{*} 0.15871, \quad \varepsilon_{\mathrm{ssdg} 8}=4.9318 \\
& \varepsilon_{\mathrm{ssdg} 9}=4.9318-\mathrm{i}^{*} 0.15565 .
\end{aligned}
$$

This combination of parameters is not exclusive. The set of parameters given by Eq. (7) is just an illustration, and this example is generated so that the values of real and imaginary parts of dielectric permittivities are within realizable limits, the condition that can obviously be satisfied by other parameters combinations. As already pointed out, some materials must have negative imaginary part of the dielectric permittivity, which categorizes them as active dielectrics $[12,13]$. In our opinion, there is an additional approach to realization of materials described by Eq. (7). It relies on (electrically or optically driven) quantum systems such as quantum cascade laser, quantum amplifier or multiple quantum wells (dots), which exhibit different values of dielectric permittivity from the background permittivity $[12,13]$. The sign and magnitude of real and imaginary part of this resultant permittivity depend on the design of the quantum structure in question (e.g. on widths of the well and the barrier layers, and on the material composition). For instance, materials with indices 3, 6 and 9 from Eq. (7) may be created so to have predefined dielectric constants at a given frequency by varying e.g. only the layer widths, within relatively narrow limits, since these permittivities are quite similar. Apparently, the same applies for group of materials with indices 1,4 , and 7 , as well as 2,5 and 8 .

Depending on the wavelength of the electromagnetic mode, the dimension of the whole structure in the $x$ direction can be varied. The minimal thickness of an 
individual layer within the generated structure is limited by the numerical step used in calculations, which is here set to $d=1 \mu \mathrm{m}$. The obtained electric field in the digitally graded structure is compared in Fig. 2 with the field of the smoothly graded structure, corresponding to the same eigenvalue. Evidently, the differences are quite small which confirms the validity of the applied approximation.

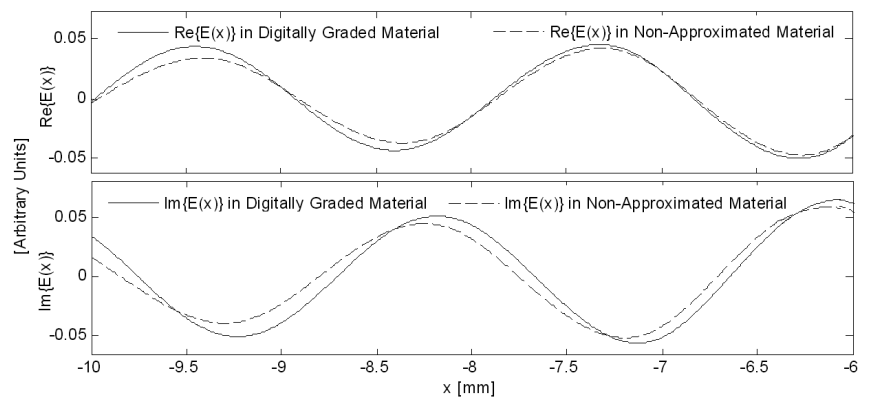

Fig. 2. Comparison of the electric fields for the initial (smoothly graded) permittivity and its digitally graded equivalent. The initial conditions are the same within the central parts of the structures, so the differences between the approximated and the "accurate" electric field functions are very small therein. The biggest difference appears at the end of the domain, which is here enlarged for improved readability.

Undoubtedly, the nature of the problem is such that it is very difficult to perform an accurate realization of the obtained results, because domain of the profile is conceptually infinite. In addition, the flattening of the peripheral areas of the potential converts the truly bound state into an oscillating one, as already pointed out. Nevertheless, the described procedure allows us to construct a digitalized structure which is experimentally realizable, and provides a satisfactory approximation to the theoretical prediction.

\section{Conclusion}

The SUSY method was used to generate complex optical potential with a localized electric field state in the continuum part of the spectrum. The obtained smooth structural profile is then processed by the digital grading technique, adapted to the case of strongly oscillating complex function of the real argument so that the structure may be realized by compiling the layers of homogeneous materials.

\section{Acknowledgments}

This work was supported by the Ministry of Science (Republic of Serbia), ev. no. 141006. V. M and J. R. also acknowledge the financial support from NATO Collaborative Linkage Grant (reference CBP.EAP.CLG 983316).

\section{References}

[1] J. von Neumann, E. Wigner, Phys. Z. 30, 465 (1929).

[2] F.H. Stillinger, D.R. Herrick, Phys. Rev. A 11, 446 (1975).

[3] F.H. Stillinger, Physica B 85, 270 (1977).

[4] D.R. Herrick, Physica B 85, 44 (1977).

[5] T.A. Weber, J. Math. Phys. 40, 140 (1999).

[6] E.N. Bulgakov, A.F. Sadreev, Phys. Rev. B 78, 075105 (2008).

[7] D.C. Marinica, A.G. Borisov, S.V. Shabanov, Phys. Rev. Lett. 100, 183902 (2008).

[8] J. Pappademos, U. Sukhatme, A. Pagnamenta, Phys. Rev. A 48, 352535 (1993).

[9] J.S. Petrović, V. Milanović, Z. Ikonić, Phys. Lett. A 300, 595 (2002).

[10] N. Prodanović, V. Milanović, J. Radovanović, J. Phys. A, Math. Theor 42, 415304 (2009).

[11] S. Vlaev, F. Garcia-Moliner, V.R. Velasco, Phys. Rev. B 52, 13784 (1995).

[12] P. Ginzburg, M. Orenstein, J. Appl. Phys. 103, 083105 (2008).

[13] P. Ginzburg, M. Orenstein, J. Appl. Phys. 104, 063513 (2008). 